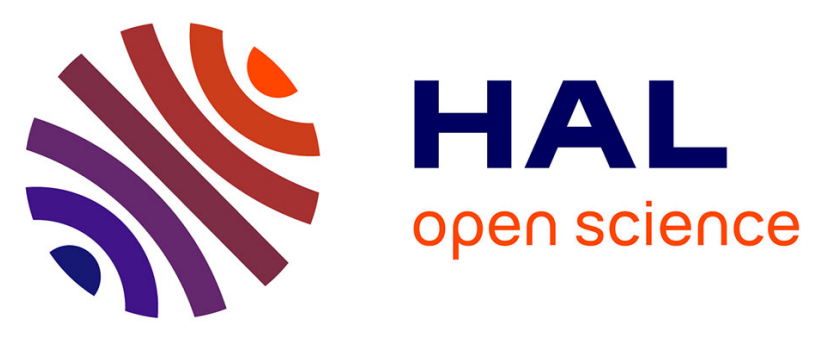

\title{
Stability of a flow down an incline with respect to two-dimensional and three-dimensional disturbances for Newtonian and non-Newtonian fluids
}

Mohamed Hatem Allouche, Séverine Millet, Valéry Botton, Daniel Henry, Hamda Ben Hadid, François Rousset

\section{To cite this version:}

Mohamed Hatem Allouche, Séverine Millet, Valéry Botton, Daniel Henry, Hamda Ben Hadid, et al.. Stability of a flow down an incline with respect to two-dimensional and three-dimensional disturbances for Newtonian and non-Newtonian fluids. Physical Review E : Statistical, Nonlinear, and Soft Matter Physics, 2015, 92 (12), pp.063010. 10.1103/PhysRevE.92.063010 . hal-01179579

\author{
HAL Id: hal-01179579 \\ https://hal.science/hal-01179579
}

Submitted on 22 Jul 2015

HAL is a multi-disciplinary open access archive for the deposit and dissemination of scientific research documents, whether they are published or not. The documents may come from teaching and research institutions in France or abroad, or from public or private research centers.
L'archive ouverte pluridisciplinaire HAL, est destinée au dépôt et à la diffusion de documents scientifiques de niveau recherche, publiés ou non, émanant des établissements d'enseignement et de recherche français ou étrangers, des laboratoires publics ou privés. 


\title{
Stability of a flow down an incline with respect to three-dimensional disturbances: the question of Squire conjecture for Newtonian or generalized Newtonian fluids
}

\author{
M.H. Allouche, S. Millet, V. Botton, D. Henry, H. Ben Hadid and F. Rousset ${ }^{1}$ \\ Laboratoire de Mécanique des Fluides et d'Acoustique, \\ CNRS/Université de Lyon, \\ École Centrale de Lyon/Université Lyon 1/INSA de Lyon, \\ ECL, 36 avenue Guy de Collongue, 69134 Ecully Cedex, France \\ ${ }^{1}$ CETHIL, UMR CNRS 5008, Université de Lyon, \\ INSA de Lyon/Université Lyon 1, \\ INSA, Bât. Sadi Carnot, 9 rue de la Physique, \\ 69621 Villeurbanne Cedex, France
}

June 23, 2015

\begin{abstract}
The Squire conjecture, which states that the two-dimensional instabilities are the more dangerous, is questioned here for a flow down an incline, making use of numerical stability analysis and Squire relationships when available. For a Newtonian fluid, it is shown that oblique wave instabilities are never the dominant instabilities. Both the Squire relationships and the particular variations of the two-dimensional wave marginal curve with regard to the inclination angle are needed to obtain this result validating the Squire conjecture. For a generalized Newtonian fluid, a similar result has only been obtained for a reduced stability problem where some term connected to the perturbation of viscosity is neglected. For the general stability problem, however, no Squire relationship can be derived and the numerical stability results show that the thresholds for oblique waves can be smaller than the thresholds for two-dimensional waves, particularly for large obliquity angles and strong non-Newtonian behaviours. The Squire conjecture is then clearly not valid in this case.
\end{abstract}




\section{Introduction}

The hydrodynamics of film flows driven by gravity down an inclined plane have been studied for a long time. Such flows, which can be encountered in many industrial and geophysical situations as well as in the everyday life, very often present intriguing wavy patterns which can become complex, depending on the slope, the flow speed and the physical properties of the fluid. The waves that are triggered in such flows are initially quasi-plane waves with a large wavelength compared to the flow depth, and they are known as surface waves. Farther downstream, the waves grow in amplitude and quickly evolve towards a non linear regime. These waves appear for non-zero Reynolds number, and have then a convective characteristic. The onset of such waves in Newtonian fluids is well understood since the early linear stability studies by Benjamin [1] and Yih [2]. A longwave approximation was adopted in these analytical approaches. They showed that the critical Reynolds number for the onset of the instabilities only depends on the inclination of the plate $\gamma$ and is proportional to $\cot \gamma$. They also pointed out that inertia is required to trigger these free surface instabilities. The experimental works of Liu [3] have confirmed this dependence with the inclination angle for the linear stability thresholds.

Most of the studies in the literature on this topic are based on a Newtonian fluid model. The rheological behavior of many fluids cannot, however, be properly described by a Newtonian model and more sophisticated rheological models involving a nonlinear relationship between stress and strain would be more appropriate. Far less studies have been carried out in the case of generalized Newtonian film flows. Ng and Mei [4] showed that a linear stability study with a power-law fluid is not sufficient to suggest a preferred wavelength for the roll wave because the predicted growth rate of the unstable disturbances increases monotonically with the wavenumber. Then, using a long-wave approximation, they demonstrated a linear evolution of the critical Reynolds number as a function of both $\cot \gamma$ and the power-law exponent $n$. This study, however, is limited by the singularity introduced by the viscosity law in the model: a power-law describes an infinite viscosity at the free surface, which is not physically consistent. To remove this singularity, some authors considered a regularized power-law model: Ruyer-Quil et al. [5] by introducing a Newtonian plateau at small strain rate and Noble and Vila [6] by introducing a weaker

formulation of the Cauchy momentum equations. They have shown the relevant influence of shear-thinning properties on the primary instability. Rousset et al. [7] studied the 
temporal stability of a Carreau fluid flow down an inclined plane. They performed an asymptotic approach considering a weakly non-Newtonian behavior in the limit of very long waves and compared it with a more general numerical approach. It was found that the critical Reynolds number is lower for shear-thinning fluids than for Newtonian fluids, while the wave celerity is larger. A particular attention was paid to the situations with small angles of inclination. Indeed, in these cases, besides the long-wave free surface mode, another instability identified by Floryan [8] as a shear mode can occur. It is characterized by a wavelength on the order of the layer thickness and a wave celerity lower than the free surface velocity. It was shown that taking into account the shear-dependence of the viscosity can change the nature of the instability.

Superposed film layers flowing down an inclined plane can be subjected to interfacial instabilities even in the limit of zero Reynolds number according to the direction of viscosity stratification. This situation was first observed with Newtonian fluids by Kao [9]. Other studies led to the same observations for non-Newtonian viscosities, such as Balmforth et al. for power-law shear-thinning fluids [10] and Herschel-Bulkley viscoplastic fluids [11] and Millet et al. [12] for Carreau shear-thinning fluids.

Most of the theoretical studies concerning flows down an incline make the assumption that the waves propagate in the same direction as the flow (waves denoted as twodimensional). Since the work of Squire [13], many people justify this simplification by saying that the two-dimensional waves are more dangerous than any oblique waves. This was in fact shown by Squire [13] for a Newtonian unidirectional forced flow between rigid boundaries. He showed that there was a relationship between the Reynolds numbers for an oblique wave and a two-dimensional wave, associated with a relationship between the wave numbers, so that the critical Reynolds number for a two-dimensional wave could be shown to be the smallest. Pearlstein [14] and Hesla et al. [15] reached the same conclusion for parallel flow of stratified Newtonian fluids. Yih [16], and, more clearly, Chang and Demekhin [17] extended these results to Newtonian flows with free-surfaces, interfaces, or density stratification. For free-surface flows down an incline, Yih [16] showed that there were also relationships between the two-dimensional and oblique wave characteristics, allowing to deduce the stability results for oblique waves from the results obtained for two-dimensional waves. These relationships, however, were more numerous and in particular included a relationship between the slopes of the inclines. This means that the Reynolds number associated with a two-dimensional wave can be shown to be smaller 
than that for an oblique wave, but this oblique wave being obtained for a larger slope. This prevents the possibility to compare directly the thresholds at a given slope. Despite this, some studies as that of Benjamin [1] use the argument of Squire [13] to justify the focus on two-dimensional instabilities. Moreover, in the case of non-Newtonian liquid film flows down an incline, Gupta and Rai [18] for visco-elastic fluids and Sahu and Matar [19] for viscoplastic fluids found that, under certain circumstances, oblique wave instabilities may be the dominant instabilities, contrary to Squire's theorem. In contrast, Nouar et al. [20] studied the three-dimensional temporal linear stability of shear-thinning fluid plane Poiseuille flows and indicated that the two-dimensional instabilities seem dominant. They, however, remark that they cannot make use of Squire relationships, which only exist for a reduced problem neglecting some terms connected with the perturbation of the viscosity and not for the general eigenvalue problem.

In this study, we focus on the flows down an incline and want to compare the thresholds of oblique waves to those of two-dimensional waves. We will consider both Newtonian and generalized Newtonian fluids (in particular Carreau fluids), with a particular distinction between the shear-thinning $(0<n<1)$ and the shear-thickening cases $(n>1), n$ being the power-law index. We will also look for the existence or not of Squire relationships, and see whether they can be used in the comparison between the thresholds associated with the different waves.

\section{Model and equations}

We want to model a film flow developing down a plate inclined by an angle $\gamma$. We will consider both Newtonian fluids with a constant viscosity $\eta_{0}$ and generalized Newtonian fluids with a viscosity $\bar{\eta}$ following the four-parameter Carreau inelastic model:

$$
\frac{\bar{\eta}-\eta_{\infty}}{\eta_{0}-\eta_{\infty}}=\left[1+(\delta \dot{\gamma})^{2}\right]^{(n-1) / 2}
$$

with $\eta_{0}$ and $\eta_{\infty}$ the limit Newtonian viscosities at low and high shear rate, respectively, $\delta$ a characteristic time, $n$ a dimensionless parameter and $\dot{\gamma}$ the local shear rate. With $0<n<1$ $(n>1)$ and $\eta_{\infty}<\eta_{0}\left(\eta_{\infty}>\eta_{0}\right)$, this law suitably describes the rheological behaviour of shear-thinning (shear-thickening) fluids. Note that the Carreau model predicts a powerlaw behaviour at moderate shear rate. However, unlike the power-law model, it predicts a viscosity that remains finite and tends to $\eta_{0}$ as the shear rate approaches zero. This

feature makes the Carreau law particularly suitable for free surface flow issues. For a 
given flow rate $Q$, the layer thickness $d$ cannot be explicitly calculated and will depend on the different Carreau law parameters: therefore, it cannot be taken as a length scale as often done. Using the dimensionless variables proposed by Weinstein [21] (length scale $d_{s}=\left(\frac{\eta_{0} Q}{\rho g \sin \gamma}\right)^{1 / 3}$, velocity scale $Q / d_{s}$, time scale $d_{s}^{2} / Q$, viscosity scale $\left.\eta_{0}\right)$, the Carreau law for the dimensionless viscosity $\eta$ becomes:

$$
\eta=I+(1-I)\left[1+\left(L \frac{d u_{b}}{d y}\right)^{2}\right]^{(n-1) / 2},
$$

where $I=\eta_{0} / \eta_{\infty}, L=\delta Q\left(\frac{\rho g \sin \gamma}{\eta_{0} Q}\right)^{2 / 3}$ and $u_{b}$ is the dimensionless basic flow velocity.

The dimensionless equations governing the flow are

$$
\left\{\begin{array}{l}
\frac{\partial u}{\partial x}+\frac{\partial v}{\partial y}+\frac{\partial w}{\partial z}=0 \\
\operatorname{Re}\left(\frac{\partial u}{\partial t}+u \frac{\partial u}{\partial x}+v \frac{\partial u}{\partial y}+w \frac{\partial u}{\partial z}\right)=-\operatorname{Re} \frac{\partial p}{\partial x}+\left(\frac{\partial \sigma_{x x}}{\partial x}+\frac{\partial \sigma_{x y}}{\partial y}+\frac{\partial \sigma_{x z}}{\partial z}\right)+1 \\
\operatorname{Re}\left(\frac{\partial v}{\partial t}+u \frac{\partial v}{\partial x}+v \frac{\partial v}{\partial y}+w \frac{\partial v}{\partial z}\right)=-\operatorname{Re} \frac{\partial p}{\partial y}+\left(\frac{\partial \sigma_{y x}}{\partial x}+\frac{\partial \sigma_{y y}}{\partial y}+\frac{\partial \sigma_{y z}}{\partial z}\right)+\cot \gamma \\
\operatorname{Re}\left(\frac{\partial w}{\partial t}+u \frac{\partial w}{\partial x}+v \frac{\partial w}{\partial y}+w \frac{\partial w}{\partial z}\right)=-\operatorname{Re} \frac{\partial p}{\partial z}+\left(\frac{\partial \sigma_{z x}}{\partial x}+\frac{\partial \sigma_{z y}}{\partial y}+\frac{\partial \sigma_{z z}}{\partial z}\right)
\end{array},\right.
$$

where $u, v$, and $w$ are the dimensionless velocities along the $x, y$, and $z$ directions, respectively, $p$ is the dimensionless pressure (dimensionalized by $\rho Q^{2} / d_{s}^{2}$ ), $\sigma_{i j}$ is the dimensionless viscous strain tensor, and Re is the Reynolds number defined by $\operatorname{Re}=\rho Q / \eta_{0}$. This system has to be solved with the associated boundary conditions at the bottom $y=d$ and at the free surface $y=0$.

The system (3) has a steady solution $u_{b}$ featuring a uniform longitudinal flow only depending on the normal direction $y$. For this basic solution, the equations are reduced to

$$
\left\{\begin{array}{l}
\frac{d \sigma_{x y}^{b}}{d y}=\frac{d}{d y}\left(\eta \frac{d u_{b}}{d y}\right)=-1 \\
\frac{d p_{b}}{d y}=\frac{\cot \gamma}{\mathrm{Re}}
\end{array},\right.
$$

and the boundary conditions correspond to no slip at the bottom and no friction stress at the flat free surface:

$$
\left\{\begin{array}{l}
u_{b}(y=d)=0 \\
\sigma_{x y}^{b}(y=0)=0
\end{array},\right.
$$

where $\sigma_{x y}^{b}=\eta \frac{d u_{b}}{d y}$ is the only non-zero component of the viscous strain tensor for the basic flow. In the general case where $\eta$ is given by the Carreau law (2), there is no analytical solution to these equations and $u_{b}$ has to be obtained numerically. 


\section{Stability analysis}

We perform a temporal linear stability study on this problem. The basic flow is perturbed by fluctuations of the velocity, $u^{\prime}, v^{\prime}$ and $w^{\prime}$ and of the pressure, $p^{\prime}$ and by the fluctuation of the interface, $\zeta^{\prime}$. We obtain $u=u_{b}(y)+u^{\prime}(x, y, z, t), v=v^{\prime}(x, y, z, t), w=w^{\prime}(x, y, z, t)$, $p=p_{b}(y)+p^{\prime}(x, y, z, t), \zeta=\zeta^{\prime}(x, z, t)$ and $\sigma_{x y}=\sigma_{x y}^{b}(y)+\sigma_{x y}^{\prime}(x, y, z, t)$. These expressions are introduced in the system (3) and after linearization, we get:

$$
\left\{\begin{array}{l}
\frac{\partial u^{\prime}}{\partial x}+\frac{\partial v^{\prime}}{\partial y}+\frac{\partial w^{\prime}}{\partial z}=0 \\
\operatorname{Re}\left(\frac{\partial u^{\prime}}{\partial t}+u_{b} \frac{\partial u^{\prime}}{\partial x}+v^{\prime} \frac{d u_{b}}{d y}\right)=-\operatorname{Re} \frac{\partial p^{\prime}}{\partial x}+\left(\frac{\partial \sigma_{x x}^{\prime}}{\partial x}+\frac{\partial \sigma_{x y}^{\prime}}{\partial y}+\frac{\partial \sigma_{x z}^{\prime}}{\partial z}\right) \\
\operatorname{Re}\left(\frac{\partial v^{\prime}}{\partial t}+u_{b} \frac{\partial v^{\prime}}{\partial x}\right)=-\operatorname{Re} \frac{\partial p^{\prime}}{\partial y}+\left(\frac{\partial \sigma_{y x}^{\prime}}{\partial x}+\frac{\partial \sigma_{y y}^{\prime}}{\partial y}+\frac{\partial \sigma_{y z}^{\prime}}{\partial z}\right) \\
\operatorname{Re}\left(\frac{\partial w^{\prime}}{\partial t}+u_{b} \frac{\partial w^{\prime}}{\partial x}\right)=-\operatorname{Re} \frac{\partial p^{\prime}}{\partial z}+\left(\frac{\partial \sigma_{z x}^{\prime}}{\partial x}+\frac{\partial \sigma_{z y}^{\prime}}{\partial y}+\frac{\partial \sigma_{z z}^{\prime}}{\partial z}\right)
\end{array}\right.
$$

where the strain perturbations are:

$$
\left\{\begin{array}{l}
\sigma_{x x}^{\prime}=2 \eta \frac{\partial u^{\prime}}{\partial x} ; \sigma_{y y}^{\prime}=2 \eta \frac{\partial v^{\prime}}{\partial y} ; \sigma_{z z}^{\prime}=2 \eta \frac{\partial w^{\prime}}{\partial z} \\
\sigma_{x z}^{\prime}=\eta\left(\frac{\partial u^{\prime}}{\partial z}+\frac{\partial w^{\prime}}{\partial x}\right) ; \sigma_{y z}^{\prime}=\eta\left(\frac{\partial v^{\prime}}{\partial z}+\frac{\partial w^{\prime}}{\partial y}\right) ; \sigma_{x y}^{\prime}=\theta\left(\frac{\partial u^{\prime}}{\partial y}+\frac{\partial v^{\prime}}{\partial x}\right)
\end{array} .\right.
$$

Note that in the expression of the perturbed viscous strain in the $(x, y)$ plane, a new viscosity $\theta$ appears $[7,20]$. This is due to the fact that, in this plane, the velocity perturbations induce viscosity perturbations. $\theta$ is given by:

$$
\theta=I+(1-I)\left[1+n\left(L \frac{d u_{b}}{d y}\right)^{2}\right]\left[1+\left(L \frac{d u_{b}}{d y}\right)^{2}\right]^{n-3 / 2}
$$

The boundary conditions associated to this perturbation problem are the no-slip condition at the bottom:

$$
u^{\prime}=v^{\prime}=w^{\prime}=0 \text { at } y=d,
$$

the kinematic condition at the free surface:

$$
v^{\prime}=\frac{\partial \zeta^{\prime}}{\partial t}+U_{b} \frac{\partial \zeta^{\prime}}{\partial x}=0 \text { at } y=0
$$

the zero viscous strain at the perturbed free surface, which gives:

$$
\left\{\begin{array}{l}
\sigma_{x y}^{\prime}-\zeta^{\prime}=0 \\
\sigma_{y z}^{\prime}=0 \\
\sigma_{x z}^{\prime}=0
\end{array} \quad \text { at } y=0,\right.
$$


and the normal strain balance at the perturbed free surface, which gives:

$$
\zeta^{\prime} \cot \gamma+\operatorname{Re} p^{\prime}-2 \eta \frac{\partial v^{\prime}}{\partial y}+\frac{1}{C a}\left(\frac{\partial^{2} \zeta^{\prime}}{\partial x^{2}}+\frac{\partial^{2} \zeta^{\prime}}{\partial z^{2}}\right)=0 \text { at } y=0
$$

where $C a=\frac{\eta_{0} Q}{\sigma d_{s}}$ is the capillary number and $\sigma$ is the surface tension.

The perturbations of velocity, $u^{\prime}, v^{\prime}$ and $w^{\prime}$ and of pressure, $p^{\prime}$ and the fluctuation of the interface $\zeta^{\prime}$ are developped as three-dimensional normal modes:

$$
\left\{\begin{array}{l}
{\left[u^{\prime}, v^{\prime}, w^{\prime}, p^{\prime}\right](x, y, z, t)=[\hat{u}, \hat{v}, \hat{w}, \hat{p}](y) e^{i(\alpha x+\beta z-\alpha c t)}} \\
\zeta^{\prime}(x, z, t)=\hat{\zeta} e^{i(\alpha x+\beta z-\alpha c t)}
\end{array}\right.
$$

where $(\hat{u}, \hat{v}, \hat{w}, \hat{p}, \hat{\zeta})$ are complex variables, $(\alpha, 0, \beta)$ is the real wave vector (with $\alpha^{2}+$ $\beta^{2}=k^{2}$ ) and $c$ is the dimensionless complex celerity of the wave. Its real part $c_{r}$ gives the dimensionless phase velocity and its imaginary part $c_{i}$ gives the time amplification rate. A stable (unstable) flow will correspond to negative (positive) values of $c_{i}$ and the perturbations will be called three-dimensional if $\beta \neq 0$ and two-dimensional if $\beta=0$. Introducing this formulation in the governing equations for the perturbations (6) and combining these equations adequately, we obtain a system of two coupled equations for the unknowns $(\hat{v}, \hat{w})$ :

$$
\begin{aligned}
& i \alpha \operatorname{Re}\left[\left(u_{b}-c\right)\left(D^{2}-k^{2}\right)-D^{2}\left(u_{b}\right)\right] \hat{v}=-4 k^{2} D(\eta D \hat{v})+ \\
& {\left[D^{2} \theta+2 D \theta D+\theta\left(D^{2}+k^{2}\right)\right]\left(D^{2}+k^{2}\right) \hat{v}+\frac{i \beta\left(D^{2}+k^{2}\right)[(\theta-\eta)(D \hat{w}+i \beta \hat{v})],}{i \alpha \operatorname{Re}\left(u_{b}-c\right)(i \beta \hat{v}-D \hat{w})-i \alpha \operatorname{Re} D u_{b} \hat{w}=\beta^{2} \theta D \hat{w}+k^{2} D(\eta \hat{w})-i \beta \theta\left(D^{2}+\alpha^{2}\right) \hat{v}+}} \\
& 3 i \beta D(\eta D \hat{v})-i \beta\left(D^{2}+\beta^{2}\right)(\eta \hat{v})-\left(D^{2}+\beta^{2}\right)(\eta D \hat{w}),
\end{aligned}
$$

where $D=\frac{d}{d y}$ is the derivative with respect to the normal direction $y$. The first equation is a generalized Orr-Sommerfeld equation for the normal velocity perturbation $\hat{v}$, which, however, cannot be solved alone in the general case due to the presence of the underlined term involving the perturbation $\hat{w}$. To obtain the boundary conditions, we must note that, at the free surface, the basic flow shear is zero $\left(D u_{b}=0\right)$, so that $\eta=\theta=1$ and $D \eta=D \theta=0$. These boundary conditions are:

$$
\begin{gathered}
\hat{v}=D \hat{v}=\hat{w}=0 \quad \text { at } y=d, \\
{\left[1+\left(u_{b}-c\right)\left(D^{2}+k^{2}\right)\right] \hat{v}=0 \quad \text { at } y=0,} \\
i \beta \hat{v}+D \hat{w}=0 \quad \text { at } y=0,
\end{gathered}
$$




$$
\begin{gathered}
-\beta D \hat{v}+i\left(\alpha^{2}-\beta^{2}\right) \hat{w}=0 \quad \text { at } y=0 \\
-i k^{2}\left(D^{2}+k^{2}\right)\left[\frac{1}{\alpha \tan \gamma}+\frac{k^{2}}{\alpha C a}\right] \hat{v}+4 k^{2} D \hat{v}+i \alpha R e\left(u_{b}-c\right) D \hat{v} \\
-D\left[\left(D^{2}+k^{2}\right) \hat{v}\right]=0 \quad \text { at } y=0 .
\end{gathered}
$$

In the case of two-dimensional perturbations $(\beta=0, k=\alpha)$, the system (14)-(15) for generalized Newtonian fluids is reduced to the equation (14) without the underlined term, i.e. a generalized Orr-Sommerfeld equation given in [7]:

$$
\begin{aligned}
i \alpha \operatorname{Re}\left[\left(u_{b}-c\right)\left(D^{2}-k^{2}\right)-D^{2}\left(u_{b}\right)\right] \hat{v} & =-4 k^{2} D(\eta D \hat{v}) \\
& +\left[D^{2} \theta+2 D \theta D+\theta\left(D^{2}+k^{2}\right)\right]\left(D^{2}+k^{2}\right) \hat{v}
\end{aligned}
$$

which, for two-dimensional perturbations, is solved with $\alpha=k$. Conversely, the threedimensional perturbations for a Newtonian fluid $(\theta=\eta=1)$ verify the usual OrrSommerfeld equation deduced from (14):

$$
i \alpha \operatorname{Re}\left[\left(u_{b}-c\right)\left(D^{2}-k^{2}\right)-D^{2}\left(u_{b}\right)\right] \hat{v}=\left(D^{2}-k^{2}\right)^{2} \hat{v} .
$$

\section{Numerical procedure}

A spectral Tau collocation method based on Chebyshev polynomials is used for the discretization of the generalized eigenvalue problem (14)-(20). The resulting system of algebraic equations, solved on the Gauss-Lobatto collocation points $\left(y_{j}=\cos (j \pi / N)\right.$ for $j=0, N)$ in the layer, can be written in the abbreviated form

$$
[A] X=\omega[B] X,
$$

where $X$ is the vector containing the algebraic values of $\hat{v}$ and $\hat{w}$ at each collocation point. The dimension of the square matrices $[A]$ and $[B]$ is twice the number of modes $N+1$. The eigenvalues obtained when solving (23) are the complex angular frequencies $\omega=\alpha c$, and the imaginary part of $\omega$ is the growth rate $\omega_{I}$.

From the spectra obtained by solving (23), we will compute neutral curves (values of Re for which an eigenmode has a zero growth rate whereas all the other eigenmodes have a negative growth rate) depending on the wave numbers $\alpha$ and $\beta$, from which critical Reynolds number $\operatorname{Re}_{c}$ can be obtained by minimization along $\alpha$ and $\beta$. The numerical procedure has been validated in previous studies [7, 12]. 


\section{Results for a Newtonian fluid}

In the case of a Newtonian fluid, the three-dimensional stability problem is governed by the Orr-Sommerfeld equation (22) and the associated boundary conditions (those on $\hat{v}$ deduced from (16), (17) and (20)). As already shown by Yih [16] and Chang and Demekhin [17], we have different relationships between the characteristics of the oblique waves and of the two-dimensional waves, which we will denote with the subscripts $3 \mathrm{D}$ and $2 \mathrm{D}$, respectively. If the wave number for the two-dimensional waves, $\alpha_{2 D}$, is directly denoted as $k\left(\alpha_{2 D}=k\right.$ in this case) and the wave numbers for the oblique waves simply as $\alpha$ and $\beta$, the different relationships are:

$$
\begin{aligned}
\alpha^{2}+\beta^{2} & =k^{2}, \\
\alpha \operatorname{Re}_{3 D} & =k \operatorname{Re}_{2 D}, \\
\alpha \tan \gamma_{3 D} & =k \tan \gamma_{2 D}, \\
\alpha \mathrm{Ca}_{3 D} & =k \mathrm{Ca}_{2 D} .
\end{aligned}
$$

Equations (24) and (25) come from the Orr-Sommerfeld equation (as for rigid boundaries), and (26) and (27) from the boundary conditions. These relationships indicate that the stability results for the oblique waves can be obtained from those for the twodimensional waves, but for different involved wall inclinations. Then we cannot easily conclude for the comparison at a given wall inclination.

It is then interesting to compare numerically the three-dimensional and the twodimensional instability thresholds. This comparison is given in figure 1 for a wall inclination $\gamma=2^{\circ}$ and $1 / \mathrm{Ca}=0$. The neutral curves, Re versus $\alpha$, have been obtained first for given values of the wave number $\beta$ (figure 1a) and then for given values of the wave obliquity angle $i_{o b}$ defined as $\tan \left(i_{o b}\right)=\beta / \alpha$ (figure 1b). The two-dimensional neutral curve decreases regularly when decreasing $\alpha$ and tends towards a minimum value when $\alpha$ becomes small. In contrast, when decreasing $\alpha$, the three-dimensional neutral curves obtained at given values of $\beta$ decrease towards a minimum reached for a finite value of $\alpha$ and then increase strongly when $\alpha$ becomes small. A decrease of $\beta$ induces a drift of the minimum towards lower values of both $\alpha$ and $R e$; the increase at small $\alpha$ is thus observed to be steeper. In any case, these neutral curves appear to be above the two-dimensional neutral curve. When obtained at given obliquity angle (figure 1b), the neutral curves look more similar to the two-dimensional curve, with a monotonous variation and a minimum 
reached asymptotically for small $\alpha$. These curves, still above the two-dimensional curve, continuously tend to this curve when the obliquity angle $i_{o b}$ is decreased to $0^{\circ}$. It is interesting to see that some properties of these three-dimensional neutral curves can be infered from the Squire relationships (24)-(27).

We will still assume for simplicity that $1 / \mathrm{Ca}=0$. If we first consider that $\beta \neq 0$ and $\alpha=0$ (i.e. $k=\beta$ ), the relation (26) gives $\gamma_{2 D}=0$. As there is no instability in the horizontal situation, $\operatorname{Re}_{2 D} \rightarrow \infty$. Finally, the relation (25) gives $\operatorname{Re}_{3 D}>>\operatorname{Re}_{2 D}$, so that $\operatorname{Re}_{3 D} \rightarrow \infty$. We then obtain that for any non-zero wave vector $\beta$, the neutral curve obtained for a given inclination $\gamma_{3 D}$ tends towards infinity when $\alpha$ decreases to zero, what is observed in figure 1a. Conversely, if we now consider that the waves have a given obliquity $i_{o b}\left(i_{o b} \neq 90^{\circ}\right)$, relation $(26)$ gives $\tan \left(\gamma_{2 D}\right)=\tan \left(\gamma_{3 D}\right) \cos \left(i_{o b}\right)$, so that $\gamma_{2 D}$ is non zero and $\operatorname{Re}_{2 D}$ is finite. Relation (25) then gives $\operatorname{Re}_{3 D}=\operatorname{Re}_{2 D} / \cos \left(i_{o b}\right)$, indicating that $\operatorname{Re}_{3 D}$ also remains finite. We then obtain that for a given obliquity $i_{o b} \neq 90^{\circ}$, the neutral curve obtained for a given inclination $\gamma_{3 D}$ remains finite when $\alpha$ decreases to zero, what is observed in figure $1 b$.

The comparison shown above seems to indicate that the three-dimensional thresholds are larger than the two-dimensional thresholds. To justify this, it is interesting to consider the two-dimensional wave stability results which are shown in figure 2 as heavy dashed lines. When the inclination of the plate $\gamma$ increases, the neutral curves (figure $2 \mathrm{a}$ ) globally decrease, as well as the minimum value $\operatorname{Re}_{c}$ reached for small $\alpha$. The stability curve showing $\operatorname{Re}_{c}$ versus $\gamma$ (figure $2 \mathrm{~b}$ ) is then a continuously decreasing curve. Returning to the Squire relationships used for $1 / \mathrm{Ca}=0$, we can write:

$$
\operatorname{Re}_{3 D}\left(\gamma_{3 D}\right)>\operatorname{Re}_{2 D}\left(\gamma_{2 D}\right)
$$

from (25) and $\gamma_{3 D}>\gamma_{2 D}$ from (26). The decrease of the curves in figure 2 then indicates that $\operatorname{Re}_{2 D}\left(\gamma_{2 D}\right)>\operatorname{Re}_{2 D}\left(\gamma_{3 D}\right)$, and the combination with (28) finally gives that

$$
\operatorname{Re}_{3 D}\left(\gamma_{3 D}\right)>\operatorname{Re}_{2 D}\left(\gamma_{3 D}\right)
$$

Using the Squire relationships together with the results obtained for two-dimensional waves, it is thus possible to show that, for a Newtonian fluid flowing down an incline, the two-dimensional waves are the more dangerous. Note that this proof uses a property of the two-dimensional stability study result, namely that $\operatorname{Re}_{2 D}\left(\gamma_{2 D}\right)$ monotonously decreases. In this case the Squire conjecture is thus validated a posteriori rather than stated prior to the two-dimensional study. 


\section{Results for a generalized Newtonian fluid}

The general system we have to solve for a generalized Newtonian fluid flowing down an incline is given by equations (14)-(15) with associated boundary conditions. As already shown, for two-dimensional waves, the system is reduced to equation (21) with $\alpha=k$. The two-dimensional wave results obtained in the shear-thinning case for $L=0.5, n=0.5$ and $I=0$ and in the shear-thickening case for $L=0.5, n=1.5$ and $I=0$ are also given in figure 2 with solid lines and dashed-dotted lines, respectively. These stability results obtained for generalized Newtonian fluids look similar to those obtained for Newtonian fluids, with similar neutral curves decreasing as a whole when $\gamma$ is increased (figure 2a) and a stability curve decreasing as well (figure $2 \mathrm{~b}$ ). All these values, however, are smaller (larger) for the shear-thinning fluid (shear-thickening fluid) than for the Newtonian fluid. The influence of $L$ on the critical Reynolds number for these generalized Newtonian fluids is shown in figure 3 for a fixed inclination angle $\gamma=2^{\circ}$. As expected, we observe a decrease of the critical Reynolds number with $L$ for the shear-thinning fluids $(0<n<1)$ and an increase for the shear-thickening fluids $(n>1)$. Note also the symmetry around the Newtonian case $(n=1)$ in the neutral curves between the shear-thickening $(n=1.2$, 1.5 and 1.65) and the shear-thinning $(n=0.8,0.5$ and 0.35$)$ cases.

Following the same assumptions as Nouar et al. [20], a simplification of the threedimensional wave problem can be obtained if we consider that $\theta=\eta$ in the underlined term in equation (14). This assumes that the perturbations of viscosity are neglected in this term, which could be thought as a weak assumption as the instability is driven by the shear at the free surface, where $\theta=\eta=1$. With this assumption, the system to solve is reduced to a single equation given by (21). For this equation and the associated boundary conditions, the same Squire relationships as in (24)-(27) can be derived, indicating that the same type of neutral curves as in figure 1 can be obtained for this three-dimensional wave reduced problem. Moreover, the Squire relationships and the decrease of the twodimensional wave stability curves (figure $2 \mathrm{~b}$ ) also indicate that, for shear-thinning fluids as well as for shear-thickening fluids, the two-dimensional wave thresholds are smaller than the three-dimensional wave thresholds obtained for this reduced problem.

It is now useful to solve the general system (14)-(15) to check if the indications obtained with the reduced problem can be confirmed or not. The neutral curves obtained for an inclination $\gamma=2^{\circ}, n=0.5, I=0$ and different obliquities of the three-dimensional waves 
are shown in figure 4 , both for the general problem (denoted as $3 D_{2}$ ) and the reduced problem (denoted as $\left.3 D_{1}\right)$. The two-dimensional results $\left(i_{o b}=0^{\circ}\right)$ are also given for comparison. Each graph corresponds to a different value of the parameter $L, L=0.5$, 0.7, 0.8, and 1. As expected, the neutral Reynolds number values obtained with the reduced problem are above those obtained in the two-dimensional case. The values for the general problem, however, are below those for the reduced problem, sometimes much below, indicating that the assumption used to reduce the three-dimensional problem is not so weak in the shear-thinning case. For small values of $L$ as $L \leq 0.7$, the two-dimensional neutral values seem to remain the smallest. In contrast, for larger values of $L$, the neutral curves of the general problem can be below the two-dimensional neutral curves, particularly for large values of the obliquity angle $i_{o b}$. The critical curves, $\operatorname{Re}_{c}$ versus $L$, obtained in the different approximations of the problem are also shown in figure 5 for different obliquity angles. In any case, the curves decrease when $L$ is increased. The critical curves for the general problem, however, decrease more rapidly than those obtained for the reduced problem. This effect is particularly important for the large obliquity angles, where the departure between the two curves corresponding to the different approximations strongly increases with $L$. As a result, the critical curves for the general problem can decrease below the two-dimensional wave curves when $L$ is increased, and this effect seems to occur at smaller $L$ values when the obliquity angle $i_{o b}$ is increased. These results thus indicate that three-dimensional wave instabilities can be the more dangerous in shearthinning fluids, particularly those with a large obliquity angle, and for sufficient values of $L$. Another conclusion is that the perturbations of viscosity cannot be neglected in this three-dimensional wave instability problem for shear-thinning fluids.

The three-dimensional wave instability curves obtained by solving either the general problem or the reduced problem in the shear-thickening case are also given in figure 6 . For the shear-thickening case, both types of critical curves increase when $L$ is increased, but the curves obtained for the general problem increase more rapidly than those obtained for the reduced problem. As it was previously shown that the instability thresholds for the reduced problem are higher than the two-dimensional thresholds, the critical curves for the general problem in the shear-thickening case will remain above the corresponding two-dimensional critical curve for all values of the obliquity angle $i_{o b}$. These results thus indicate that, in the shear-thickening case, the oblique wave instabilities are never the dominant instabilities for any value of $L$, even if the Squire relationships cannot be derived 
for the general problem in this case.

\section{Conclusion}

This study has been focused on the possible occurence of three-dimensional wave instabilities as the dominant instability in flows down an incline. The two cases of Newtonian fluids and generalized Newtonian fluids have been considered. For Newtonian fluids, it was possible to show that the three-dimensional wave instabilities are never the dominant instabilities, so that the well known long wavelength free-surface two-dimensional waves remain the more dangerous. This result cannot be obtained from the Squire relationships alone, but need to make use of the particular variation of the two-dimensional critical curve with regard to the inclination angle. The result was further confirmed by some three-dimensional wave stability results. In contrast, for generalized Newtonian fluids, Squire relationships only exist for a reduced problem neglecting some terms connected with the perturbation of the viscosity and not for the general problem. For this reduced problem, we can still conclude that the long wavelength free-surface two-dimensional waves are the more dangerous. For the general problem, however, no conclusion can be obtained from Squire considerations as Squire relationships cannot be derived for these generalized Newtonian fluids. Nevertheless, some numerical stability calculations have shown that the thresholds for oblique waves can be smaller than the thresholds for two-dimensional waves, particularly for large obliquity angles and strong shear-thinning properties, whereas the thresholds for oblique waves are always higher than the two-dimensional thresholds for shear-thickening fluids.

In conclusion, the Squire conjecture, which says that the two-dimensional instabilities are the more dangerous and is known to be valid in a channel flow of Newtonian fluid, has been shown to remain valid for a flow down an incline for a Newtonian fluid. It seems to be also valid for shear-thickening fluids. In contrast, for shear-thinning fluids, this conjecture is no more valid, as cases have been found where the oblique waves are the more dangerous instabilities. 


\section{References}

[1] T.B. Benjamin. Wave formation in laminar flow down an inclined plane. Journal of Fluid Mechanics, 2(06):554-573, 1957.

[2] C.-S. Yih. Stability of liquid flow down an inclined plane. Physics of Fluids, 6(3):321$334,1963$.

[3] J. Liu, J.D. Paul, and J.P. Gollub. Measurements of the primary instabilities of film flows. Journal of Fluid Mechanics, 250:69-101, 1993.

[4] C.-O. Ng and C. C. Mei. Roll Waves on a Shallow Layer of Mud Modeled as a Power-Law Fluid. Journal of Fluid Mechanics, 263:151-183, 1994.

[5] C. Ruyer-Quil, S. Chakraborty, and B. S. Dandapat. Wavy Regime of a Power-Law Film Flow. Journal of Fluid Mechanics, 692:220-256, 2012.

[6] P. Noble and J.-P. Vila. Thin power-law film flow down an inclined plane: consistent shallow-water models and stability under large-scale perturbations. Journal of Fluid Mechanics, 735:29-60, 2013.

[7] F. Rousset, S. Millet, V. Botton, and H. Ben Hadid. Temporal stability of Carreau fluid flow down an incline. Journal of Fluids Engineering - transactions of the ASME, 129(7):913-920, 2007.

[8] J.M. Floryan, S.H. Davis, and R.E. Kelly. Instabilities of a liquid film flowing down a slightly inclined plane. Physics of Fluids, 30:983-989, 1987.

[9] T. W. Kao. Role of viscosity stratification in the stability of two-layer flow down an incline. Journal of Fluid Mechanics, 33:561-572, 1968.

[10] N. J. Balmforth, R. V. Craster, and C. Toniolo. Interfacial instability in nonNewtonian fluid layers. Physics of Fluids, 15(11):3370-3384, 2003.

[11] N. J. Balmforth and J. J. Liu. Roll waves in mud. Journal of Fluid Mechanics, 519:33-54, 2004.

[12] S. Millet, V. Botton, H. Ben Hadid, D. Henry, and F. Rousset. Stability of two-layer shear-thinning film flows. Phys. Rev. E, 88:043004, 2013. 
[13] H.B. Squire. On the stability for three-dimensional disturbances of viscous fluid flow between parallel walls. In Proceedings of the Royal Society A: Mathematical, Physical and Engineering Sciences, volume 142, pages 621-628, 1933.

[14] A. J. Pearlstein. On the two-dimensionality of the critical disturbances for stratified viscous plane parallel shear flows. Physics of Fluids, 28(2):751-753, 1985.

[15] T. I. Hesla, F. R. Pranckh, and L. Preziosi. Squire's theorem for two stratified fluids. Physics of Fluids, 29(9):2808-2811, 1986.

[16] C.-S. Yih. Stability of two-dimensional parallel flows for three-dimensional disturbances. Quarterly of Applied Mathematics, 12(4):434-435, 1955.

[17] H.C. Chang and E.A. Demekhin. Complex wave dynamics on thin films. Elsevier, 2002.

[18] A.S. Gupta and L. Rai. Note on the stability of a visco-elastic liquid film flowing down an inclined plane. Journal of Fluid Mechanics, 33(01):87-91, 1968.

[19] K. C. Sahu and O. K. Matar. Three-dimensional linear instability in pressure-driven two-layer channel flow of a Newtonian and a Herschel-Bulkley fluid. Physics of Fluids, 22(11):112103, 2010.

[20] C. Nouar, A. Bottaro, and J.P. Brancher. Delaying transition to turbulence in channel flow: revisiting the stability of shear-thinning fluids. Journal of Fluid Mechanics, 592:177-194, 2007.

[21] S.J. Weinstein. Wave propagation in the flow of shear-thinning fluids down an incline. AIChE Journal, 36(12):1873-1889, 1990. 

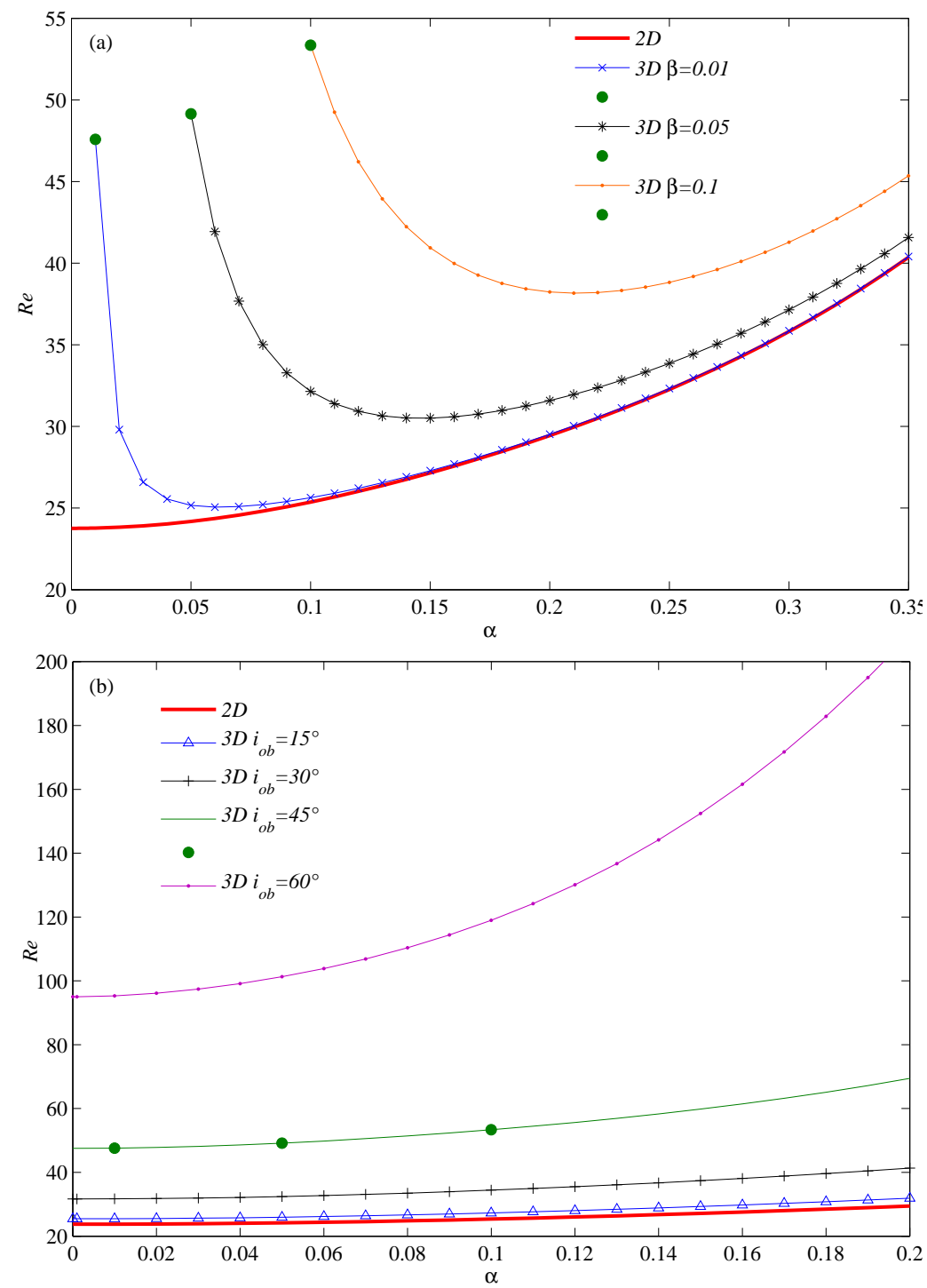

Figure 1: Three-dimensional wave stability results for the flow down an incline in the case of a Newtonian fluid and for a fixed inclination of the plate $\gamma=2^{\circ}(1 / \mathrm{Ca}=0)$. The neutral curves, expressed as the Reynolds number Re versus the streamwise wave number $\alpha$, are given for different values of the transverse wave number $\beta$ (a), or for different obliquities of the waves $i_{o b}$ (b). In (a), for each value of $\beta$, the points corresponding to $\alpha=\beta$ are indicated. These points correspond to those shown in (b) for $i_{o b}=45^{\circ}$, showing the correspondence between the two plots. The two-dimensional wave neutral curve is given as a red heavy solid line for comparison. 

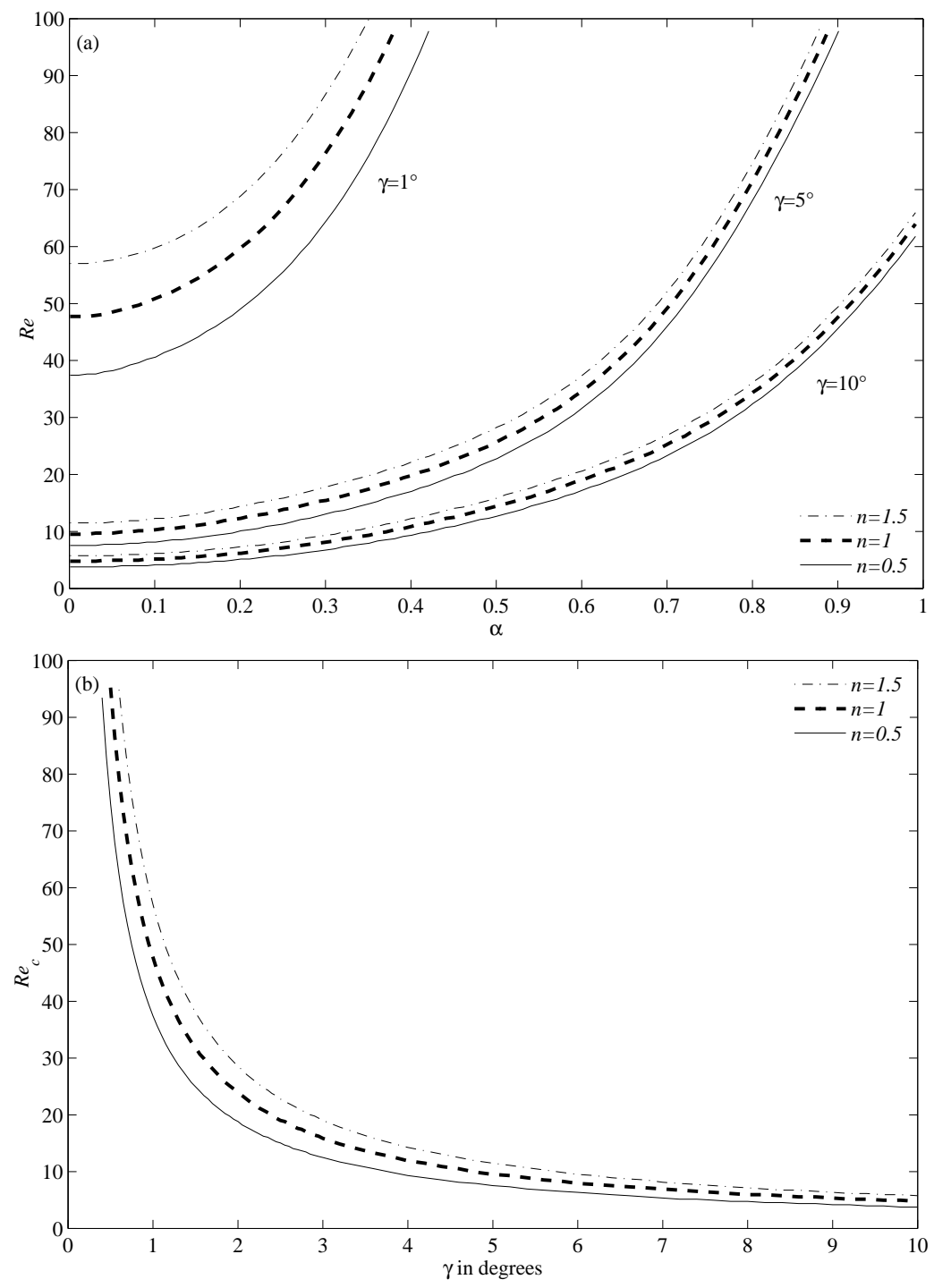

Figure 2: Two-dimensional wave stability results for the flow down an incline in the case of generalized Newtonian fluids $(1 / \mathrm{Ca}=0)$ : (a) neutral curves expressed as the Reynolds number Re versus the streamwise wave number $\alpha$ for different inclinations $\gamma$ of the plate; (b) stability curve showing the critical Reynolds number $\operatorname{Re}_{c}$ as a function of the inclination $\gamma$. In (a) and (b), the cases corresponding to a Newtonian fluid (heavy dashed lines) are compared to those corresponding to a shear-thinning fluid $(L=0.5, n=0.5, I=0)$ (solid lines) and those corresponding to a shear-thickening fluid ( $L=0.5, n=1.5, I=0$ ) (dotted-dashed lines). 


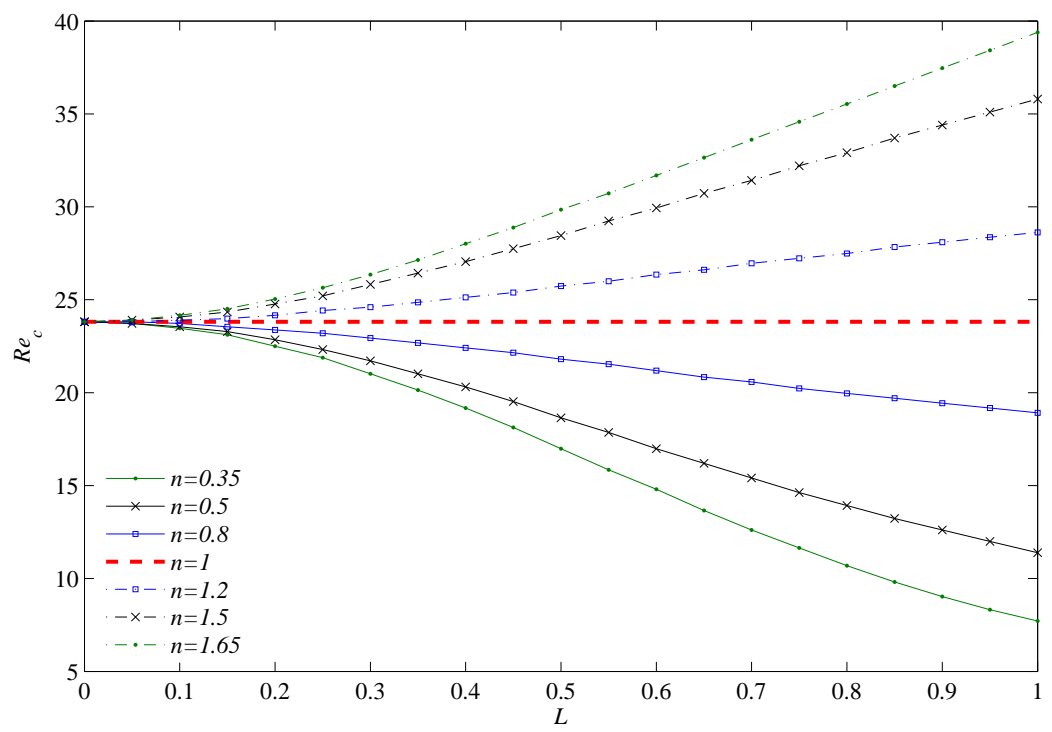

Figure 3: Two-dimensional wave stability results for the flow down an incline for a fixed inclination of the plate, $\gamma=2^{\circ}(I=0,1 / C a=0)$ : stability curves showing the critical Reynolds number $R e_{c}$ as a function of $L$. The results are obtained for different values of the power-law index for shear-thinning (solid lines) and shear-thickening (dotted-dashed lines) fluids. The constant value obtained in the Newtonian case is given as a red heavy dashed line for comparison. 

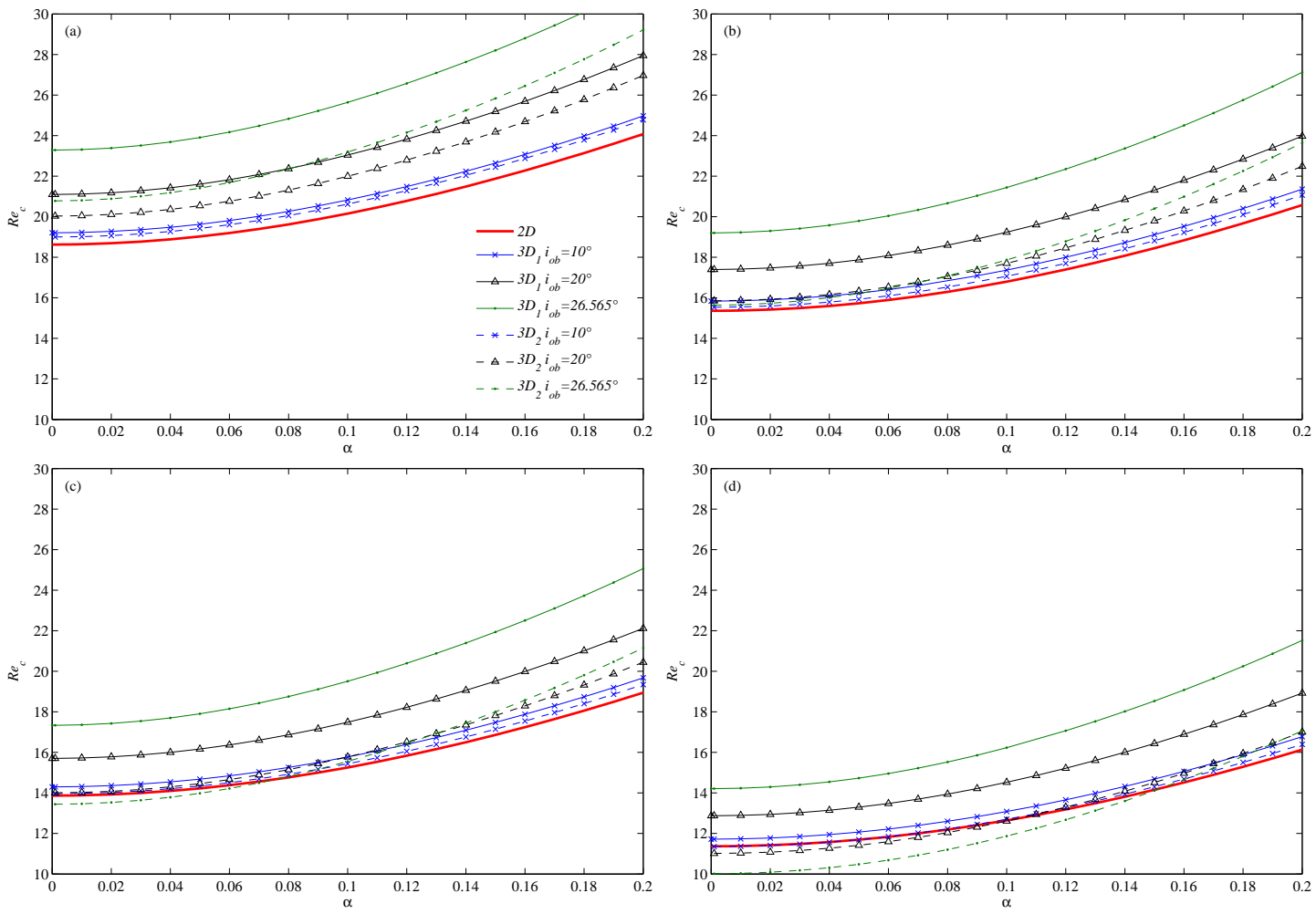

Figure 4: Three-dimensional wave stability results for the flow down an incline in the case of a shear-thinning fluid and for a fixed inclination of the plate, $\gamma=2^{\circ}(n=0.5$, $I=0,1 / \mathrm{Ca}=0)$. The neutral curves, expressed as the Reynolds number Re versus the streamwise wave number $\alpha$, are given for different obliquities of the wave $i_{o b}$ and for different values of $L, L=0.5$ (a), $L=0.7$ (b), $L=0.8$ (c) and $L=1$ (d). The results obtained in the general case $\left(3 D_{2}\right.$, dashed lines) are compared with those obtained with the reduced model ( $3 D_{1}$, solid lines). The curve for $i_{o b}=26.565^{\circ}$ corresponds to $\beta=\alpha / 2$. For each value of $L$, the two-dimensional wave neutral curve is given as a red heavy solid line for comparison. 


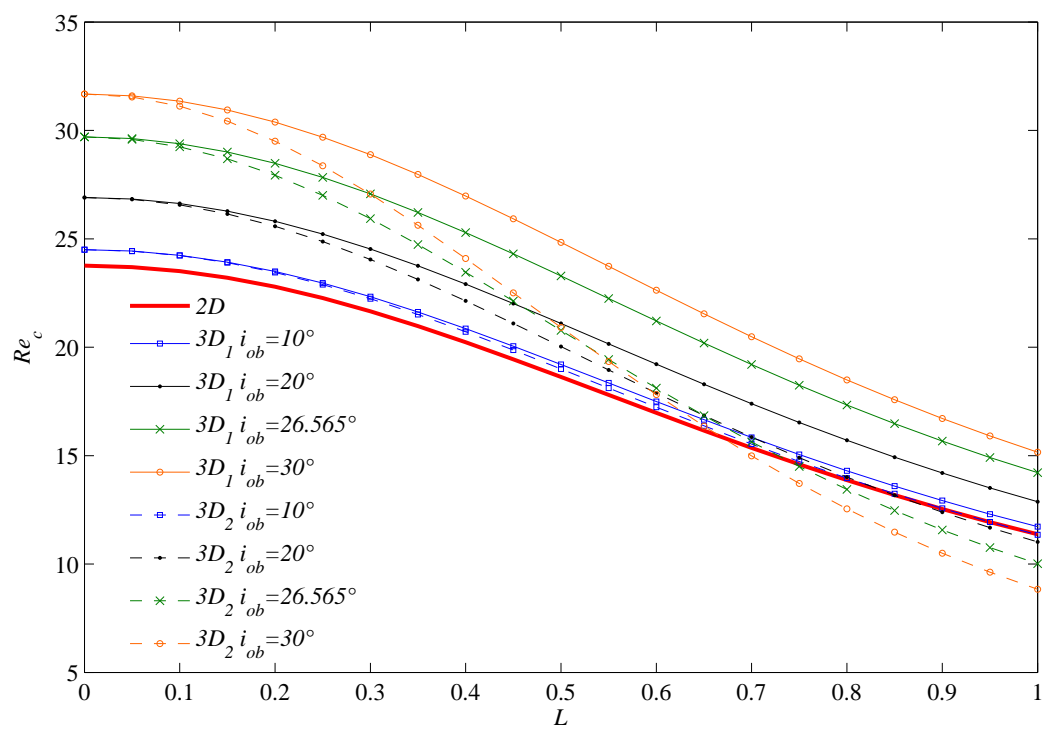

Figure 5: Three-dimensional wave stability results for the flow down an incline in the case of a shear-thinning fluid and for a fixed inclination of the plate, $\gamma=2^{\circ}(n=0.5, I=0$, $1 / \mathrm{Ca}=0)$ : stability curves showing the critical Reynolds number $\operatorname{Re}_{c}$ as a function of $L$. The results are obtained for different obliquities of the waves (from $i_{o b}=0$ to $30^{\circ}$ ), for the general problem $\left(3 D_{2}\right.$, dashed lines) and for the reduced model $\left(3 D_{1}\right.$, solid lines). The two-dimensional wave stability results are given as a red heavy solid line for comparison. 


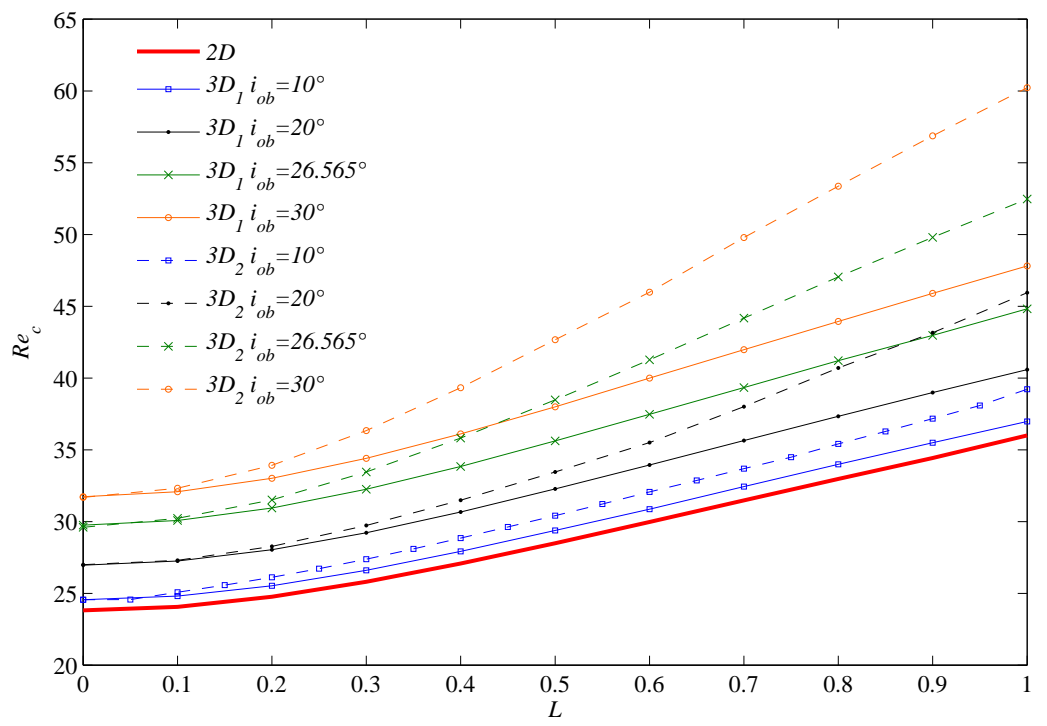

Figure 6: Three-dimensional wave stability results for the flow down an incline in the case of a shear-thickening fluid and for a fixed inclination of the plate, $\gamma=2^{\circ}(n=1.5, I=0$, $1 / \mathrm{Ca}=0)$ : stability curves showing the critical Reynolds number $\operatorname{Re}_{c}$ as a function of $L$. The results are obtained for different obliquities of the waves (from $i_{o b}=0$ to $30^{\circ}$ ), for the general problem $\left(3 D_{2}\right.$, dashed lines) and for the reduced model ( $3 D_{1}$, solid lines). The two-dimensional wave stability results are given as a red heavy solid line for comparison. 